\title{
Rhythm control strategy in the transcatheter ablation era
}

\author{
Riccardo Maddalena \\ Cardiology Unit, Fabrizio Spaziani Hospital, Frosinone, Italy
}

Received: 2021-05-22.

Accepted: 2021-06-13

This work is licensed under a Creative Commons Attribution 4.0 International License

J Clin Med Kaz 2021; 18(4):12-15

Corresponding author:

Riccardo Maddalena.

E-mail: riccardo.maddalena@alice.it

\begin{abstract}
The latest ESC guidelines on atrial fibrillation limit rhythm control to symptomatic cases, continuing on the same line that the Affirm and Race trials traced twenty years ago. There is evidence though that rhythm control, net of the adverse effects of the antiarrhythmic drugs, is useful to improve the prognosis beyond just the reduction of symptoms. With transcatheter ablation we can now finally split the clinical benefit of an antiarrhythmic therapy from the negative impact on prognosis of the medical approach, especially in patients with structural heart disease. We must ask ourselves nowadays, in light of the latest trials analyzed in this review, if there is space for a first line rhythm control strategy, if it should be pursued regardless of symptoms, with what modalities and how much in particular the patient with reduced ejection fraction can benefit from it.
\end{abstract}

Key words: atrial fibrillation, rhythm control, ablation

\section{A double edged weapon}

Atria are physiologically responsible for one third of the diastolic ventricular filling and the importance of their mechanical function is bigger in patients with intermediate grades of diastolic dysfunction, in whom the atrial kick is compensatory to the reduced ventricular compliance in order to maintain a valid preload. From these premises it comes natural to consider useful a rhythm control strategy in most of the AF patients, making the atria return to their physiological condition. For decades, however, data suggested the opposite, indicating the simple rate control as non inferior or even better than rhythm control in term of adverse reactions and hospitalizations. On this basis, guidelines recommend sinus rhythm restoration only in AF patients remaining symptomatic despite rate control. Antiarrhythmic drugs have always represented a double edged weapon. If on the one hand they've been shown to prevent arrhythmias, on the other they've sometimes increased all cause mortality and death from arrhythmias. Some of them combine arrhythmogenicity and non cardiac adverse reactions with the antiarrhythmic effect. The CAST trial showed that sodium current inhibition can increase mortality in patients with ischemic cardiopathy [1] and, seven years later, the SWORD trial showed that $\mathrm{IKr}$ inhibition can also be dangerous in patients with ischemic cardiopathy and systolic dysfunction [2]. Dronedarone can double mortality in patients with HFrEF NYHA III-IV (ANDROMEDA trial) [3] and in patients with permanent AF (PALLAS trial) [4]. A recent metanalysis by Valembois et al. have shown that sotalol can double the risk of death in patients with atrial fibrillation (NNH 102) [5]. Sotalol use for long-term rhythm control, once recommended as class I, is now a class IIb recommendation. The AFFIRM trial, twenty years ago, showed a trend for increased mortality with rhythm control compared to rate control (HR 1,15 95\% CI 0,99-1,34), as well as a significantly greater number of hospitalizations, on a sample of more than 4000 patients with AF (one third of which at the first episode) and a mean LVEF 55\% [6]. A subsequent analysis of this trial clearly showed the double face of the antiarrhythmic drugs, which have a useful antiarrhythmic effect but also have a dangerous effect, which increases the risk of adverse clinical events. An as treated analysis of the AFFIRM, after considering the antiarrhythmic therapy and the sinus rhythm as different covariates, revealed that the antiarrhythmic therapy increases significantly the risk of death. Significance which was concealed if sinus rhythm was removed from the multivariate analysis. The rhythm control, even if by drugs, seems then useful clinically, but this usefulness is actually hidden by the other adverse effects that drugs lead to. This concealment seems to be greater in patients with normal systolic function, as outlined by the subgroup analysis of the AFFIRM trial. Also in the RACE, this time in patients with persistent AF only, the rate control approached superiority for the primary efficacy endpoint (HR 0,73 90\% CI 0,53-1,01) [7]. It must be said that, in both the trials, anticoagulation therapy was sometimes stopped after some weeks of effective rhythm control, which led to a better rate control than in the rate control arm itself, but which was obtained in a small percentage of patients $(62 \%$ after 5 years in the AFFIRM and 39\% at the end of follow up in the RACE trial). 


\section{AF in patients with reduced systolic function}

The better performance of antiarrhythmic drugs in patients with reduced systolic function, as outlined by the subgroup analysis of the AFFIRM, led some years later to the AF-CHF, a trial on 1367 AF patients with EF $\leq 35 \%$ NYHA II-IV, with a 37 months follow up, which did not show significant differences between rate and rhythm control in the primary endpoint of cardiovascular death (HR 1,06 95\% CI 0,86-1,3), [8] but did not show either a trend for greater risk of death or ictus in the rhythm control arm, against what the AFFIRM suggested, according to the concept that a ventricle with systolic dysfunction benefits more from a preserved atrial function. With this in mind, transcatheter ablation of atrial fibrillation, devoid of the clinical adverse effects of antiarrhythmic drugs, can further enhance the benefits of rhythm control in patients with systolic dysfunction. This is what emerged from the ARC-HF trial, on a small sample of patients with persistent AF and severely depressed systolic function $(24 \pm 8 \%)$, in which transcatheter ablation significantly increased the VO2 max during a CPET, a prognostic marker of long term survival, and decreased the left atrium area, compared to a rate control strategy [9]. The exercise tolerance developed progressively during the follow up, indicating a resultant negative ventricular remodeling after sinus rhythm restoration. Similar benefits have been reached in the CAMTAF trial, on patients with persistent $\mathrm{AF}$ and reduced $\mathrm{EF}$, in which ablation significantly increased LVEF compared to rate control $(8,1 \%$ vs $-3,6 \% \mathrm{p}<0,001)$ during a six months follow up, with a negative $\mathrm{LV}$ remodeling totally absent in the rate control arm $(\triangle \mathrm{LVESV}$ $-14,2 \%$ vs $4,7 \% \mathrm{p}=0,03$ ), in which a positive remodelling has been noted instead [10]. This was the first trial to show ablation superiority, compared to rate control, even in patients with systolic dysfunction and asymptomatic AF. According to these results, transcatheter ablation resulted superior than amiodarone in the AATAC trial, in patients with persistent AF and systolic dysfunction, both in reducing relapses (70\% vs $34 \%$ in sinus rhythm after 2 years, $95 \%$ CI $25-44 \%, \mathrm{p}<0,001)$ and in decreasing mortality $(8 \%$ vs $18 \% \mathrm{p}=0,037)$ or hospitalization for $\mathrm{AF}$ or heart failure ( $31 \%$ vs $57 \%$ p $<0,001$ ), with a low NNT moreover (10 for mortality and 3,8 for hospitalizations), even if the trial was not designed to test such endpoints [11]. The superiority of ablation in reducing relapses, compared to antiarrhythmic drugs, was already been shown by the MANTRA PAF trial in patients with preserved ejection fraction [12].

Regarding hard clinical endpoints like death or hospitalizations for heart failure, in 2018 the CASTLE-AF trial, on a sample of 363 patients with symptomatic AF and $\mathrm{LVEF}<35 \%$, showed that ablation is superior than standard medical therapy, which consisted of rate control associated with antiarrhythmic drugs in $30 \%$ of patients [13]. Ablation reduced by $38 \%$ the risk of death or HF hospitalization compared to medical therapy (HR 0,62 95\% CI 0,43-0,87), with a NNT of only 8 and similar NNT for each component of the composite outcome. The reduction of all cause death became significant after 3 years of follow up, according to the concept that rhythm control has a positive effect on ventricular remodeling and on the clinical history of heart failure patients. It must be said that the CASTLE recruited patients unsuccessfully treated with antiarrhythmic drugs, selecting a subgroup partially resistant to a rhythm control approach and making it impossible to draw conclusions about a first line ablative approach, which may be even more useful in more responsive patients. In the subgroup analysis of the CASTLE it came out that a severely depressed systolic function $(\mathrm{EF}<25 \%)$ affects significantly ( $\mathrm{p}$ value for Journal of Clinical Medicine of Kazakhstan: 2021 Volume 18, Issue 4
Figure 1 - Algorithm for AF rhythm control in the transcatheter ablation era

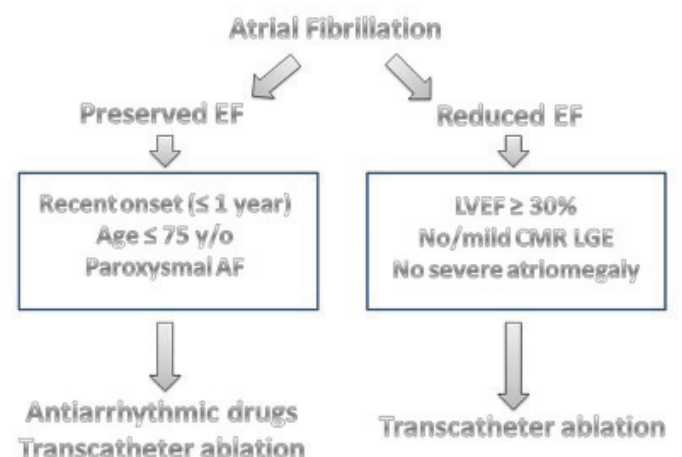

interaction $=0,01)$ the benefit of ablation compared to medical therapy (HR 1,36 95\% CI 0,69-2,65). The little effectiveness of ablation in patients with severely impaired systolic function has been confirmed later by the AMICA trial, in which ablation did not improved EF compared to optimal medical therapy ( $\triangle \mathrm{LVEF}$ $8,8 \%$ vs $7,3 \% \mathrm{p}=0,36)$ on a sample of 140 patients with non paroxysmal AF and a median EF of $27,6 \%$ (compared to $32,5 \%$ in CASTLE-AF), even if rhythm control was more efficacious in the ablation arm $(73,5 \%$ of patients in sinus rhythm after 1 years compared to $50 \%$ in the OMT arm) [14]. In the CASTLE, ablation was compared to a control arm in which rhythm control was possible by drugs. We should ask ourselves if ablation would be also superior, in patients with systolic dysfunction, to rate control only. It's what shown by the CAMERA MRI trial indeed, which recruited patients with tachycardiomyopathy (LVEF $\leq 45 \%$ ) due to persistent AF [15]. In these patients, ablation significantly increased EF compared to the rate control arm $(+18 \pm 13 \%$ vs $+4,4 \pm 13 \%$ after 6 months, $p<0,0001)$, with smaller advantages if MRI showed ventricular late enhancement after gadolinium injection. These data, besides those showed by the AMICA trial, suggest that the more a ventricle is remodeled and structurally altered, the less it benefits from a restored atrial function. A rhythm control strategy is therefore the more effective in heart failure the more $\mathrm{AF}$ is responsible for the failure itself. The CAMERA MRI trial showed that AF, which increases the risk of death, ictus and HF progression in patients with reduced systolic function, does not find in the increased heart rate the only mechanism for worsening the prognosis. In fact, the ablation arm achieved superiority over the primary efficacy endpoint despite a good rate control in the rate control arm. Transcatheter ablation, net of the adverse effects of the antiarrhythmic drugs in patients with structural heart disease, not only decreases heart rate, but also resolves the irregularity of the ventricular response and it restores the presystolic filling due to the atrial contraction, two factors promoting ventricular remodeling when altered. If the CASTLE trial compared ablation with medical therapy (which included rhythm control by drugs in one third of the patients), the RAFT-AF, currently in progress, is comparing ablation therapy with the rate control only on a sample of 411 patients with high burden AF and heart failure NYHA II-III, on a primary outcome composite of death and heart failure exacerbation [16]. The CONTRA-HF will evaluate the benefits of cryoablation compared to optimal medical therapy in patients with severe heart failure [17].

\section{AF in patients with preserved systolic function}

We should ask if ablation may also be useful in symptomatic AF patients with preserved systolic function. The CABANA trial compared ablation with medical therapy (rhythm or rate 
control) on a sample of 2204 patients, with a EF greater than $35 \%$ in more than $95 \%$ of cases, on a follow up of five years [18]. In the intention to treat analysis, ablation did not achieve superiority for the primary efficacy outcome, probably due to the low frequency of events and a crossover as high as $30 \%$ from the medical therapy to the ablation arm, but it achieved superiority in the as treated analysis (HR $0,67 \mathrm{p}=0,006$ ), which is not altered by crossover, with a significant reduction of all cause death (HR 0,6 p=0,005). In the intention to treat analysis, by the way, ablation significantly reduced cardiovascular death and hospitalizations (HR 0,83 p=0,001). The EAST AFNET 4 trial, in the transcatheter ablation era, showed that a rhythm control strategy may be better than a rate control one, despite what the AFFIRM showed two decades ago [19]. The EAST studied a sample of 2789 patients under $75 \mathrm{y} / \mathrm{o}$, with AF since less than one year, mostly with preserved systolic function, during a follow up of five years, comparing an early rhythm control strategy (both by ablation or by drugs) with usual therapy (rhythm control in symptomatic patients only). Early rhythm control achieved superiority in the primary composite outcome of cardiovascular death, stroke and hospitalizations for cardiovascular causes (HR $0,79 p=0,005)$, even if there was a NNT of just 91 . There were no differences between the arms in the nights spent in the hospital or in the systolic function changes. In the subgroup analysis of the EAST, the early rhythm control strategy was superior in patients with heart failure too.

\section{Conclusion}

In conclusion, nowadays, the early rhythm control seems prognostically useful in patients with impaired systolic function, probably also in asymptomatic ones, preferably by transcatheter ablation, which not so infrequently has to be redone a second time. Out of this rule there are patients with severely impaired systolic function or with marked structural alterations of the ventricles, in which remodeling progression has gone too far to benefit from a restored sinus rhythm, and patients with severe atriomegaly (left atrium anteroposterior diameter greater than $6 \mathrm{~cm}$ ), excluded from the most of the aforementioned trials, in which a rhythm control strategy would be of little benefit cause of a high number of relapses. An early rhythm control strategy should also be considered, according to the EAST-AFNET 4 trial, in asymptomatic patients younger than 75 with recent onset AF (diagnosed since less than one year), whatever the systolic function. We still have to understand, in these patients, if a transcatheter ablation should be done as a first line step or after an ineffective attempt at rhythm control by drugs. The ATHENA and the PALLAS trials showed that a rhythm control strategy by drugs can be clinically useful, decreasing hard endpoints like cardiovascular death, if patients have a recent onset AF, mostly in sinus rhythm (ATHENA) [20], being harmful instead, doubling mortality, in patients with AF since more than two years or with long-standing persistent AF (PALLAS). Finally, we still have to understand the optimal transcatheter ablation strategy, beyond the simple pulmonary vein isolation.

Disclosures: There is no conflict of interest for all authors.

Acknowledgements: None

Funding: None

\section{References}

1. Debra S. Echt, Philip R. Liebson, Brent Mitchell, Robert W. Peters, Dulce Obias-Manno, Allan H. Barker et al. Mortality and Morbidity in Patients Receiving Encainide, Flecainide, or Placebo - The Cardiac Arrhythmia Suppression Trial. N Eng J Med. 1991; 324:781-788. DOI: 10.1056/NEJM199103213241201

2. Albert L Waldo, A J Camm, H deRuyter, P L Friedman, D J MacNeil, J F Paulus et al. Effect of d-sotalol on mortality in patients with left ventricular dysfunction after recent and remote myocardial infarction. The Lancet. 1996; 348:7-12. DOI:https://doi.org/10.1016/ S0140-6736(96)02149-6

3. Lars Køber, Christian Torp-Pedersen, John J V McMurray, Ole Gøtzsche,Samuel Lévy, Harry Crijns et al. Increased Mortality after Dronedarone Therapy for Severe Heart Failure. N Eng J Med. 2008; 358:2678-2687. DOI: 10.1056/NEJMoa0800456

4. Stuart J. Connolly, A. John Camm, Jonathan L. Halperin, Campbell Joyner, Marco Aligns, John Amerena et al. Dronedarone in HighRisk Permanent Atrial Fibrillation. N Eng J Med. 2011; 365:2268-2276. DOI: 10.1056/NEJMoa1109867

5. Lucie Valembois, Etienne Audureau, Andrea Takeda, Witold Jarzebowski, Joel Belmin, Carmelo Lafuente-Lafuente. Antiarrhythmics for maintaining sinus thythm after cardioversion of atrial fibrillation. Cochrane Database Syst Rev. 2019. doi: 10.1002/14651858. CD005049.pub5

6. The AFFIRM Investigators. A comparison of rate control and rhythm control in patients with atrial fibrillation. $N$ Eng $J$ Med. 2002; 347:1825-1833. DOI: 10.1056/NEJMoa021328

7. Isabelle C Van Gelder, Vincent E Hagens, Hans A Bosker, J Herre Kingma, Otto Kamp, Tsjerk Kingma et al. A comparison of rate control and rhythm control in patients with recurrent persistent atrial fibrillation. $N$ Eng J Med. 2002; 347:1834-40. DOI: 10.1056/ NEJMoa021328

8. Denis Roy, Mario Talajic, Stanley Nattel, George Wyse, Paul Dorian, Kerry L. Lee et al. Rhythm Control versus Rate Control for Atrial Fibrillation and Heart Failure. N Eng J Med. 2008; 358:2667-2677. DOI: 10.1056/NEJMoa0708789

9. David G Jones, Shouvik K Haldar, Wajid Hussain, Rakesh Sharma, Darrel P Francis, Shelley L Rahman-Haley et al. A randomized trial to assess catheter ablation versus rate control in the management of persistent atrial fibrillation in heart failure. J Am Coll Cardiol. 2013; 61:1894-1903. DOI: 10.1016/j.jacc.2013.01.069. Epub 2013 Mar 7

10. Ross J Hunter, Thomas J Berriman, Ihab Diab, Ravindu Kamdar, Laura Richmond, Victoria Baker et al. A randomized controlled trial of catheter ablation versus medical treatment of atrial fibrillation in heart failure (the CAMTAF trial). Circ Arrhythm Electrophysiol. 2014; 7:31-38. DOI: 10.1161/CIRCEP.113.000806. Epub 2014 Jan 1

11. Luigi Di Biase, Prasant Mohanty, Sanghamitra Mohanty, Pasquale Santangeli, Chintan Trivedi, Dhanunjaya Lakkireddy et al. Ablation Versus Amiodarone for Treatment of Persistent Atrial Fibrillation in Patients With Congestive Heart Failure and an Implanted Device: Results From the AATAC Multicenter Randomized Trial. Circulation. 2016; 133:1637-44. DOI: 10.1161/ CIRCULATIONAHA.115.019406. Epub 2016 Mar 30 
12. Jens Cosedis Nielsen, Arne Johannessen, Pekka Raatikainen, Gerhard Hindricks, Hakan Walfridsson, Ole Kongstad et al. Radiofrequency Ablation as Initial Therapy in Paroxysmal Atrial Fibrillation. N Eng J Med. 2012; 367:1587-1595. DOI: 10.1056/NEJMoa1113566

13. 13. Nassir F. Marrouche, Johannes Brachmann, Dietrich Andresen, Jurgen Siebels, Lucas Boersma, Luc Jordaens et al. Catheter Ablation for Atrial Fibrillation with Heart Failure. N Eng J Med. 2018; 378:417-427. DOI: 10.1056/NEJMoa1707855

14. Karl-Heinz Kuck, Bela Merkely, Ralf Zahn, Thomas Arentz, Karlheinz Seidl, Michael Schluter et al. Catheter Ablation Versus Best Medical Therapy in Patients With Persistent Atrial Fibrillation and Congestive Heart Failure: The Randomized AMICA Trial. Circ Arrhythm Electrophysiol. 2019; 12. DOI: 10.1161/CIRCEP.119.007731. Epub 2019 Nov 25

15. Sandeep Prabhu, Andrew J Taylor, Ben T Costello, David M Kaye, Alex J A McLellan, Aleksandr Voskoboinik et al. Catheter Ablation Versus Medical Rate Control in Atrial Fibrillation and Systolic Dysfunction: The CAMERA-MRI Study. J Am Coll Cardiol. 2017; 70:1949-1961. DOI: 10.1016/j.jacc.2017.08.041. Epub 2017 Aug 27

16. Rhythm Control - Catheter Ablation With or Without Antiarrhythmic Drug Control of Maintaining Sinus Rhythm Versus Rate Control With Medical Therapy and/or Atrio-ventricular Junction Ablation and Pacemaker Treatment for Atrial Fibrillation (RAFT-AF). ClinicalTrials.gov NCT01420393

17. Ablation of Atrial Fibrillation in Heart Failure Patients (CONTRA-HF). ClinicalTrials.gov NCT03062241

18. Douglas L. Packer, Daniel B. Mark, Richard A. Robb, Kristi H. Monahan, Tristram D. Bahnson, Jeanne E. Poole et al. Effect of Catheter Ablation vs Antiarrhythmic Drug Therapy on Mortality, Stroke, Bleeding, and Cardiac Arrest Among Patients With Atrial Fibrillation The CABANA Randomized Cliical Trial. JAMA. 2019; 321:1261-1274. DOI: 10.1001/jama.2019.0693

19. Paulus Kirchhof, A. John Camm, Andreas Goette, Axel Brandes, Lars Eckardt, Arif Elvan et al. Early Rhythm-Control Therapy in Patients with Atrial Fibrillation. N Eng J Med. 2020; 383:1305-1316. DOI: 10.1056/NEJMoa2019422. Epub 2020 Aug 29

20. Stefan H. Hohnloser, Harry J.G.M. Crijns, Martin van Eickels, Christophe Gaudin, Richard L. Page, Christian Torp.Pedersen et al. Effect of Dronedarone on Cardiovascular Events in Atrial Fibrillation. N Eng J Med. 2009; 360:668-78. DOI: 10.1056/NEJMoa0803778 